\title{
Terapia Ocupacional em reabilitação na Atenção Primária à Saúde: possibilidades e desafios
}

\section{Occupational Therapy in rehabilitation in Primary Health Care: challenges and opportunities}

\author{
Eucenir Fredini Rocha ${ }^{1}$, Camila Cristina Bortolozzo \\ Ximenes de Souza ${ }^{2}$
}

\begin{abstract}
ROCHA, E. F.; SOUZA, C. C. B. X. Terapia Ocupacional em reabilitação na Atenção Primária à Saúde: possibilidades e desafios. Rev. Ter. Ocup. Univ. São Paulo, v. 22, n. 1, p. 36-44, jan./ abr. 2011.

RESUMO: O objetivo deste artigo é contribuir com reflexões para consolidar ações que a Terapia Ocupacional (TO) pode desenvolver na Atenção Primária em Saúde (APS) no campo da reabilitação de pessoas com deficiências. Trata-se de uma proposição fundamentada em revisão bibliográfica da literatura e em experiências práticas do REATA- Laboratório de Estudos em Reabilitação e Tecnologia Assistiva no período de 1999/2010. Foram considerados os modelos assistenciais vigentes, a APS Seletiva e Ampliada, a constituição do conjunto dessas práticas no contexto histórico e prático-organizacional. Em seguida, são descritas as experiências desenvolvidas pelo REATA na APS, que motivaram a apresentação dessas reflexões em forma de proposição do trabalho da TO. Defendeu-se a necessidade de um deslocamento das intervenções terapêuticas ocupacionais instituídas sob vertentes normatizadoras, biomédicas, ou edificadas na concepção de saúde pública de base higienista, para a constituição de ações/estratégias que contemplem aspectos holísticos dos sujeitos e do território, com tecnologias leves e adequadas às demandas, como previstos na concepção de uma APS Ampliada. Finalmente, apresentaram-se possíveis contribuições da TO no que se refere às suas potencialidades tecnológicas a partir da premissa que as ações dessa profissão podem contribuir para a promoção do acesso e da longitudinalidade na assistência em saúde e dessa forma colaborar na efetivação da integralidade e equidade no SUS.
\end{abstract}

DESCRITORES: Terapia ocupacional; Atenção primária à saúde; Saúde da família; Núcleo familiar; Reabilitação; Pessoas com deficiência/reabilitação.

\footnotetext{
1. Mestranda do Departamento de Medicina Preventiva da Faculdade de Medicina da Universidade de São Paulo e Terapeuta Ocupacional do Departamento de Fisioterapia, Fonoaudiologia e Terapia Ocupacional da Faculdade de Medicina da Universidade de São Paulo.

2. Prof $^{\mathrm{a}} \mathrm{Dr}^{\mathrm{a}}$ do Departamento de Fisioterapia, Fonoaudiologia e Terapia Ocupacional da Faculdade de Medicina da Universidade de São Paulo.

Endereço para correspondência: Rua Cipotânea, no 51 - Cidade Universitária. CEP. 05360-160 - São Paulo, SP - e-mail: eucenir@usp.br
} 


\section{APRESENTAÇÃO}

$\mathrm{D}$ esde a segunda metade da década de 90 as políticas de saúde em diversos países, têm demonstrado crescente preocupação com a estruturação e ampliação da Atenção Primária à Saúde (APS). No Brasil a APS tem sido objeto de diversos estudos, programas e incentivos governamentais, e novas tecnologias e campos de saberes têm sido incorporados nesse nível de atenção à saúde (GIOVANELLA; MENDONÇA, 2008).

É somente em 2008, que a Terapia Ocupacional (TO) foi considerada como uma das profissões que compõe equipes dos Núcleos de Apoio à Saúde da Família (NASF), cujo objetivo é ampliar a abrangência e o escopo das ações da APS e resolubilidade da Estratégia de Saúde da Família (ESF) (BRASIL, 2008).

No entanto, a presença da TO nesse nível assistencial é datada desde os anos 1990, quando passou a fazer parte de equipes em Unidades Básicas de Saúde (UBS), em diferentes municípios como São Paulo/SP, Belo Horizonte/ MG, Camaragibe/PE, Sobral/CE, atendendo pessoas com deficiências e incapacidades, entre outros agravos de saúde, embora na portaria do NASF a profissão seja citada somente nas práticas de saúde mental, assim se faz necessárias alterações para incluir esse profissional nas ações de reabilitação.

A bibliografia narra um esforço por parte de muitos profissionais em afirmar a inserção da profissão nas ações de reabilitação na APS, a partir de um deslocamento epistemológico de bases organicistas para intervenções dirigidas a aspectos sócio-participativos, independência e autonomia de pessoas e de grupos e constituição de redes sociais de apoio (ROCHA; KRETZER, 2008).

Atualmente, com a implantação dos NASF está ocorrendo uma ampliação da inserção da profissão nesse nível assistencial, e é necessária uma articulação dos fundamentos das práticas da TO com os princípios da APS.

\section{Experiências do REATA- Laboratório de Estu- dos em Reabilitação e Tecnologia Assistiva ${ }^{1}$ na Atenção Primária à Saúde}

O REATA está desenvolvendo atividades de ensino, extensão e pesquisa na APS desde 1999, quando foi convidado pela Secretaria de Estado de Saúde de São Paulo (SES/ $\mathrm{SP})$ para elaborar um projeto de ações de reabilitação na
ESF que foi implantado em nove UBS da região sudeste do município de São Paulo, coordenado pela Fundação Zerbini, e para vinte e seis UBS da região leste, no Programa da Saúde Família (PSF) da Casa de Saúde Santa Marcelina. Nos anos de 2000 e 2001 o laboratório coordenou a implantação do projeto em conjunto com a SES/SP e técnicos da Fundação Zerbini e Casa de Saúde Santa Marcelina, e desenvolveu atividade de supervisão técnica para as duas equipes (ROCHA; KRETZER, 2009).

Desde 2001, o laboratório oferece estágio na APS para estudantes de graduação em TO. Entre 2001 e 2002 eles ocorreram nas duas regiões do município, de 2003 a 2008 na região sudeste e de 2009 até a presente data, está sendo oferecido na UBS São Jorge, região oeste do município de São Paulo.

No campo das pesquisas na APS/ESF, o laboratório realizou vários estudos como o levantamento censitário da prevalência de pessoas com deficiências e incapacidades na região sudeste; acesso às ações de reabilitação no PSF/Fundação Zerbini; incorporação da TO no PSF; os sentidos do cuidado oferecido às pessoas com deficiência; as atribuições dos profissionais da equipe de reabilitação no PSF; avaliação da implantação das ações de reabilitação no PSF da Fundação Zerbini e Secretaria Municipal de Saúde de São Paulo no período 2000/2006 (ROCHA; KRETZER, 2008).

Da consolidação das experiências didática, assistencial, de assessoria e de pesquisa surgiram questões e propostas de organização do trabalho da TO na APS, orientadas pela efetivação dos princípios da garantia do acesso e da longitudinalidade.

\section{propostas}

Atenção Primária à Saúde: contextos,

Vários estudos têm demonstrado que os sistemas de saúde orientados pela APS, como nos países europeus, são resolutivos e apresentam maior eficiência, com impactos positivos nos indicadores de saúde. No entanto, nem sempre a APS é valorizada como o alicerce de um sistema de saúde eficaz, pois vigoram diferentes concepções a seu respeito e conseqüentemente sua organização se dá de maneiras distintas (STARFIELD, 2002; MELLO, 2009).

Em 1973, a Organização Mundial de Saúde (OMS), manifesta uma crítica aos modelos assistenciais em saúde pautados na sua organização pelas ações verticais,

\footnotetext{
1. Do curso de Terapia Ocupacional do Departamento de Fisioterapia, Fonoaudiologia e Terapia Ocupacional da Faculdade de Medicina da USP.
} 
especializadas e centradas no saber biomédico e propõe abordagens alicerçadas nos determinantes sociais do processo saúde-doença, com tecnologias adequadas às necessidades gerais de saúde da população e com elevada relação custo-benefício.

Essas proposições foram sancionadas pela Conferência de Alma-Ata em 1978, que defendeu que a APS deveria ser a primeira instância da atenção dentro de sistemas de saúde que desenvolvesse um processo de permanente assistência sanitária, com acesso pleno de toda a população, democratização de conhecimentos e inclusão de saberes populares nas práticas de saúde.

Nesse projeto de assistência sanitária, se inserem as ações de prevenção, de promoção da saúde, de cura e reabilitação, além de ações intersetoriais e educativas, ou seja, uma atuação centrada no princípio da Vigilância em Saúde (PAIM; ALMEIDA FILHO, 1998).

No entanto, no contexto internacional, houve reações à Declaração de Alma-Ata, com argumentos de que suas propostas eram muito amplas e pouco operacionais. Agências internacionais, como a Fundação Rockefeller, Banco Mundial, a Fundação Ford, o Centro Internacional para a Pesquisa e o Desenvolvimento (Canadá) e Agencia Internacional para o Desenvolvimento (USA), propuseram, em 1979, uma nova definição de APS, denominada de APS Seletiva, com propostas de intervenções de baixo custo para combater as principais doenças dos países pobres (CUETO, 2004).

De maneira geral, a cesta básica dessa proposta era composta por ações consideradas de objetivos claros, fáceis de avaliar e de aferir os resultados, como o acompanhamento do crescimento e desenvolvimento infantil, reidratação oral, aleitamento materno, imunização, suplementação alimentar, alfabetização feminina e planejamento familiar.

Esse modelo de APS prevaleceu em muitos países, orientou políticas e formação de recursos humanos nos anos subseqüentes à sua formulação. Todavia essa abordagem também sofre críticas, pois sua forma reducionista de sanar os problemas de saúde deixa muito a desejar na efetividade dos cuidados e prevenção de doenças (GIL, 2006).

Assim, a Organização Pan-Americana de Saúde (OPAS), em 2005, em consonância com proposições da OMS publicou documento em que defende a renovação da APS nas Américas (OPAS/OMS, 2005), de modo a se realizar uma atenção universal por meio de uma abordagem integral e horizontal nos sistemas de saúde, com cuidados guiados pela qualidade e com destaque na promoção, prevenção, intersetorialidade, participação social e responsabilização dos governos, que passou a ser denominada de $A P S$ Abrangente (GIOVANELLA; MENDONÇA, 2008).
Como atributos constitutivos da APS Abrangente foram definidos quatro eixos: 1. Porta de entrada: seria considerado o serviço de primeiro contato do usuário com o sistema de saúde, tanto em casos de doenças, como em cuidados gerais da saúde e encaminhamento para serviços especializados; 2. Longitudinalidade: refere-se à continuidade do acompanhamento do usuário, de um território adstrito, através de relações de cuidado e responsabilidade, ou seja, com vínculo; 3. Integralidade/Abrangência: oferta de um conjunto de intervenções que contempla aspectos orgânicos, psíquicos e sociais da saúde, reconhecendo assim a complexidade do processo saúde-doença. Essa complexidade requer também articulações nos diferentes níveis de atenção e de ações, como exames laboratoriais, especialistas, internações e articulações intersetoriais e sociais; 4. Coordenação: de cuidados na unidade de APS e nas diversas outras ações necessárias para a solução de demandas menos habituais e mais complexas dos usuários.

\section{Básica}

\section{Atenção Primária à Saúde no Brasil: Atenção}

No Brasil, a APS é atualmente nomeada pelo Ministério da Saúde (MS) como Atenção Básica. A adesão oficial à mesma data da década de 1970 , em consonância com o movimento internacional da APS (GIOVANELA; MENDONÇA, 2008).

Nesse contexto, através da Reforma Sanitária brasileira, os serviços básicos foram reorganizados e reassegurados pelo Sistema Único de Saúde (SUS), através da Constituição de 1988, que afirmou os princípios e diretrizes da universalidade, descentralização, integralidade da atenção, resolutividade, humanização do atendimento e participação social na atenção à saúde (ESCOREL, 1999).

A denominação Atenção Básica $(A B)$ teve como objetivo realizar um contraponto ao contexto histórico internacional que previa a difusão da proposta da APS Seletiva, o que desfavorece a implementação da universalidade e integralidade do SUS (GIOVANELA; MENDONÇA, 2008; MELO, 2009).

Em 2006, o MS lançou a Política Nacional de Atenção Básica que definiu e demarcou a finalidade e compreensão da APS brasileira, congregando características da $A P S$ Abrangente. Assim, $\mathrm{AB}$ tem como princípios gerais:

...um conjunto de ações de saúde, no âmbito individual e coletivo, que abrangem a promoção e a proteção da saúde, a prevenção de agravos, o diagnóstico, o tratamento, a reabilitação e a manutenção da saúde... (BRASIL, 2006, $\mathrm{p}: 10)$ 
Essa Política considera a ESF como a estratégia prioritária para a organização da $\mathrm{AB}$, explicita e almeja que esses serviços sejam a porta de entrada preferencial no sistema. No entanto, essa possibilidade não foi estabelecida como obrigatória.

Os objetivos da ESF são a humanização das práticas de saúde, o estreitamento das relações entre os trabalhadores da saúde e entre os profissionais e a comunidade, a democratização na socialização dos fatores que constituem o processo saúde-doença, o estimulo na participação da população no controle social dos serviços de saúde, a família como foco da intervenção, a realização das ações educativas de promoção e prevenção, além da assistência curativa e reabilitadora (BRASIL, 2006).

O discurso oficial da ESF pretende suplantar a visão de APS Seletiva e o modelo hospitalocêntrico, através da reorganização do trabalho que prevê o rompimento com a dicotomia entre as ações de saúde pública e de atenção médica e individual, e de levantar indicadores epidemiológicos e socioeconômicos.

A efetivação da APS Abrangente no Brasil tem sido difícil, pois são constantes as pressões das agências internacionais que patrocinam a proposta da APS Seletiva. Nesse contexto, a reorganização do sistema de saúde e do modelo assistencial, de forma a contemplar uma integração efetiva da rede de serviços, a redução das desigualdades sociais e regionais e o efetivo acesso ao SUS, ficam constantemente ameaçados (GIOVANELLA; MENDONÇA, 2008).

$\mathrm{Na}$ implantação do trabalho em equipe na ESF, ainda predomina uma abordagem biomédica, com muito despreparado para percepção das questões sociais no processo de intervenção. Na prática são priorizadas as ações de promoção, proteção e recuperação da saúde dos indivíduos e da família, do recém-nascido ao idoso, sadios ou doentes, o controle da tuberculose, a eliminação da hanseníase, controle da hipertensão, do diabetes mellitus e ações de saúde bucal, o que legitima a análise de muitos autores de que, na prática, a ESF ainda está presa aos princípios da APS Seletiva e tem dificuldades de trabalhar com o princípio da Vigilância em Saúde (MELO, 2009, p.129).

A criação dos NASF é uma das iniciativas do MS na direção de afirmar os princípios de uma APS Ampliada, e devem ser constituídos por equipes de profissionais de diferentes áreas para atuarem em parceria com as equipes de saúde da família. Segundo a portaria ministerial, eles não podem ser a porta de entrada do sistema, mas sim, devem atuar em conjunto com as equipes de saúde da família na responsabilização pelos usuários, através de acompanhamento longitudinal, com ações que favoreçam a integralidade no cuidado.

Os NASF têm a missão de desenvolver diferentes propostas, agrupados em núcleos de ação, entre elas as de Reabilitação, que tem como objetivo geral a redução de incapacidades e deficiências e reinserção social da população com esses agravos de saúde, o qual deve ser atingido através de diferentes estratégias como as ações clínicas, preventivas, educativas e de articulação intersetorial.

No entanto, apesar das diretrizes apontadas pelo MS, a eficaz implantação das ações de reabilitação nos NASF deve enfrentar pelo menos duas dificuldades: 1. o desconhecimento por parte dos gestores e profissionais de saúde, das possibilidades que as ações de reabilitação têm na APS, pois, elas, historicamente, estão inseridas no nível secundário e terciário de atenção; 2. a maioria dos profissionais de reabilitação tem uma educação acadêmica fundamentada em princípios epistemológicos de base organicista e dirigida para ações que privilegiam os procedimentos individuais, realizados em serviços de saúde especializados (ROCHA; KRETZER, 2008).

Ou seja, a primeira dificuldade está relacionada com um modelo de oferta e organização de serviços, enquanto que a segunda refere-se a uma escolha epistêmica no contexto da formação, e produzem problemas na demarcação das possibilidades da Terapia Ocupacional nesse nível assistencial.

Instigados pelas experiências concretizadas pelo REATA e à luz das reflexões sobre APS é que esse artigo prossegue, com o objetivo de vislumbrar como a Terapia Ocupacional pode compor, com o seu conjunto de saberes e práticas, o campo epistêmico e de intervenção da APS. Entende-se aqui a APS como Ampliada aquela erigida sob a perspectiva da Saúde Coletiva.

\section{Terapia Ocupacional em reabilitação na Atenção Primária em Saúde: possibilidades, desafios}

Saúde Coletiva como definido por Paim e Almeida Filho (1998) é um movimento ideológico que está comprometido com a mudança social, que supõe a articulação com novos paradigmas científicos aptos a abordar o objeto "promoção-saúde-doença-cuidado" em sua historicidade, contemplando a integralidade, produzida por diferentes saberes e práticas acerca do objeto "saúde" e que se compõe a partir de diversas disciplinas que contemplam e explicam o mesmo fenômeno, com posições distintas e observadas a partir de ângulos diversos. A proposta é de superação do biologismo dominante, da naturalização da vida social, da submissão à clínica e da dependência ao 
modelo médico hegemônico.

Para esses autores novos aspectos passam a ser considerados na redefinição do objeto de intervenção da saúde pública, ou seja, o sujeito coletivo abordado até então passa a ser compreendido a partir de novos olhares. Aspectos sociais, psicológicos, culturais são agregados na compreensão do fenômeno então denominado "processo saúde-doença". Emergem conceitos de corpo e sujeito social, grupos e classes sociais, de relações sociais, propondo a superação de abordagens que consideram apenas os indivíduos, os corpos biológicos no processo saúde-doença.

A interdisciplinaridade fica, assim, pontuada como uma demarcação necessária, pois várias disciplinas científicas são convocadas para a releitura das práticas na Saúde Pública, que necessitam se fundamentar na transdisciplinaridade, multiprofissionalidade, com ações interinstitucionais e transetoriais.

Considerando que o eixo de nossa reflexão está fundado nos paradigmas da Saúde Coletiva, entrevimos uma TO no campo da reabilitação de pessoas com deficiência e/ou incapacidades na $A P S$, cujo objeto de intervenção não é a patologia/deficiência, mas sim uma população constituída de sujeitos inseridos em uma comunidade, com os seus familiares e amigos, em atividades sociais, escolares, de trabalho, de lazer, de esporte e de cultura, pertencentes a diferentes classes sociais. Avaliam-se, ainda, nessa conformação, questões relacionadas ao estigma e ao preconceito que permeiam a condição da pessoa com deficiência e/ou incapacidades na sociedade atual.

Assim, a atenção da intervenção fica focada nas singularidades e necessidades das pessoas assistidas e nas relações interpessoais, que também são consideradas como parte do cuidado, pois é através destas, que os bons e maus encontros se materializam.

Aqui, a finalidade das ações da TO, desloca-se da intervenção voltada à correção corporal para desenvolver uma proposta dirigida a problemas coletivos e individuais de redução de incapacidades e deficiências, de melhora da qualidade de vida desse grupo populacional, de favorecimento da participação social, da constituição das redes sociais de apoio e da eliminação de preconceitos, discriminações, exclusão social e segregação.

É através do entrelaçamento das ações de promoção da saúde, prevenção de agravos, tratamento e reabilitação de deficiências e incapacidades e outros sofrimentos associados, que a integralidade, a equidade e universalização da atenção a essa população, no SUS, poderão advir e consolidar.

Portanto, de acordo com essa perspectiva, as práticas da TO na APS necessitam considerar, na sua organização e concretização, os atributos que afiançam a eficácia desse nível de atenção: a garantia do acesso e da longitudinalidade na assistência, desenvolvidos através de trabalho em equipe.

\section{Acesso aos serviços de saúde e as ações de Terapia Ocupacional}

A população com deficiência e/ou incapacidades, historicamente, tem dificuldades de acesso aos serviços de saúde, pois, tradicionalmente, é considerada como usuária dos serviços especializados (ROCHA, 2006).

Na APS, ainda poucas possibilidades assistenciais são oferecidas a essa população, pois a sua assistência não é uma ocorrência corriqueira, seja porque muitos profissionais e usuários evidenciam estranhamento com esse convívio, quer pelo despreparo técnico das equipes ou porque não possuem, na maioria dos serviços, profissionais de reabilitação que poderiam facilitar essa abordagem.

O acesso pleno da população com deficiência e/ ou incapacidades pressupõe serviços organizados para recebê-la; a articulação de ações intersetoriais que facilitem a chegada desses usuários aos mesmos e um modelo assistencial centrando em princípio ético pautado na proposta de humanização do SUS e dirigido à inclusão e participação social de todas as pessoas.

Pode-se afirmar, portanto, que as ações de TO na APS promovem a chegada e permanência dessa população, ao possibilitar a expressão de outras necessidades de assistência e contribuir na sua efetivação como porta preferencial de entrada no SUS, assegurando a universalização do acesso.

A sinalização para a população que o usuário com deficiência e/ou incapacidades pode e deve utilizar a APS traduz-se na oferta de ações específicas de TO como facilitação da constituição de ambientes domiciliares mais confortáveis, promoção de independência nas Atividades de Vida Diária, inclusão escolar e no trabalho, fomento de participações em grupos, oficinas e outras atividades coletivas, desenvolvimento de atividades de educação e apoio de cuidadores domésticos e sociais e acesso a equipamentos de Tecnologia Assistiva.

Outra frente de trabalho facilitadora do acesso são as ações que podem ser compartilhadas com outros profissionais, como atenção para situações de risco/ vulnerabilidade; implementação de ações de promoção da saúde e prevenção de agravos; educação em saúde; estudo do território; identificação do público prioritário a cada uma das ações desenvolvidas; acolhimento das demandas das equipes e matriciamento; planejamento, acompanhamento dos 
atendimentos domiciliares; fomentação da intersetorialidade; gestão do serviço; estimulação da participação dos usuários nas decisões; divulgação do trabalho de reabilitação junto à comunidade e estabelecimento de parcerias com outros níveis de atenção em saúde.

Do ponto de vista das áreas estratégicas do $\mathrm{MS}^{2}$ várias são as possibilidades de trabalho com o objetivo de detectar e intervir precocemente em alterações da funcionalidade. São ações de acompanhamento de gestantes e bebês de risco; orientações e estimulação de crianças com alterações no desenvolvimento neuro-psico-motor; organização de grupos de atividades corporais ou de outra natureza destinado às gestantes, idosos, diabéticos e hipertensos; detecção e intervenção precoce em casos de hanseníase; orientações aos cirurgiões-dentistas no atendimento de usuários com alterações neuro-motoras, como adequação postural na cadeira odontológica; atividades educativas dirigidas à população e aos profissionais de saúde, como por exemplo em temas relacionados à autonomia e independência das pessoas com deficiências no cotidiano.

Para a efetivação dessas ações é necessário que o terapeuta ocupacional tenha uma formação acadêmica que o introduza nos temas e procedimentos da saúde coletiva como atividades educativas, preventivas e de articulação intersetorial, e que domine técnicas terapêuticas ocupacionais destinadas à população com deficiências e incapacidades como manipulação corporal, adequação postural em cadeira, habilidades em transferências posturais, trabalhos corporais diversos, comunicação alternativa e/ou suplementar, domínio de fundamentos e modos operacionais de confecção de órteses e adaptações simples e de baixo custo, estimulação de bebes e crianças em risco ou com alterações no desenvolvimento, além de conhecimento de técnicas grupais, de trabalho com famílias e de manejo de situações de vulnerabilidade.

Do mesmo modo, os espaços institucionais necessitam se preparar para abrigar os procedimentos, como a disponibilização de salas para atendimento grupal e individual, com acessibilidade física e mobiliário adequado.

Para proporcionar qualidade nas intervenções é necessário disponibilizar materiais e equipamentos como colchonetes, bolas Bobath, termomoldáveis, materiais de papelaria, tintas, fios, argilas, massas, madeira, brinquedos, entre outros. Esses materiais e equipamentos devem ser encarados pelos gestores como infra-estrutura fundamental para uma ação da TO, assim como são necessários os medicamentos e outros materiais e equipamentos utilizados pelos profissionais médicos e de enfermagem.

Por fim, para assistir os usuários é necessário que a organização do serviço pressuponha a inserção no planejamento institucional de ações específicas de TO e outras compartilhadas com outros profissionais, expressas através de agenda e arranjos no cotidiano.

A TO tem importância fundamental no trabalho de articulação de ações intersetoriais a fim de constituir possibilidades efetivas de participação social, de desmonte dos processos de segregação e de exclusão social, fomentando processos que visem à melhora da urbanização dos bairros, vagas inclusivas nas escolas, acesso ao Benefício de Prestação Continuada, possibilidades alternativas de trabalho, linhas de ônibus acessíveis, enfrentamento de preconceitos, discriminações, estigmas, entre outras tantas necessidades da população com deficiência, são fundamentais no trabalho da APS.

A evidência de que muitas das necessidades dessa população não podem ser satisfeitas apenas com a ação de um profissional ou só com os serviços de saúde, impõe por si mesma, o trabalho intersetorial como necessidade.

Portanto, o compromisso ético de desenvolver um modelo assistencial dirigido para a autonomia dos sujeitos e para a participação social, como preconizado pela $A P S$ Ampliada, requer ações que privilegiem a história, o modo de vida das pessoas e a responsabilização por parte dos usuários e dos profissionais pelo processo de intervenção. Cabe ressaltar ainda que a hierarquização e regionalização não podem ser utilizadas como instrumento de transferência de responsabilidades entre os diferentes níveis assistenciais, e que a introdução de tecnologias adequadas à demanda promove resolubilidade no sistema de saúde (BUENO; MERHY, 1997).

\section{Longitudinalidade, cuidados em saúde, trabalho em equipe}

Cunha (2009) coloca que a longitudinalidade, também denominada na literatura especializada de continuidade do cuidado, é o atributo da APS considerada como central, pois é responsável pelo acompanhamento do usuário ao longo do tempo e que quando considerada

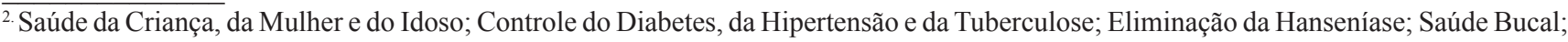
Eliminação da desnutrição infantil; Promoção da saúde.
} 
em sua plenitude impacta nas condições gerais de saúde da população e é responsável pelos resultados positivos desse nível de atenção. A autora afirma que para haver continuidade do cuidado se faz necessário o acesso ao serviço de saúde e, uma vez acessado o atendimento, a coordenação do mesmo é um agente facilitador. Lembra ainda que diferentes autores na literatura nacional e internacional diferenciam longitudinalidade de continuidade.

Longitudinalidade é o acompanhamento dos distintos problemas de saúde por um conjunto de profissionais da UBS e a continuidade refere-se ao acompanhamento de um mesmo problema por uma mesma equipe ou não, dependendo apenas de um bom sistema informacional, como os registros em prontuários e, portanto, não é um elemento constitutivo da APS (STARFIELD, 2002).

$\mathrm{O}$ atributo da longitudinalidade pode ser observado a partir de três dimensões: 1. Identificação ou reconhecimento da fonte regular de cuidados; 2. Relação interpessoal; 3 . Continuidade informacional.

Do ponto de vista da primeira dimensão, a UBS/ ESF possui adscrição de clientela e pode ser reconhecida como local de atenção dos problemas de saúde e de reabilitação das pessoas com deficiências, onde é possível abordar questões relacionadas ao conjunto do processo saúde-doença, independência e autonomia no cotidiano, participação social, desmonte de processos de segregação e exclusão social.

Em relação à segunda dimensão - relações interpessoais - a qualidade do atendimento terapêutico ocupacional, a assiduidade nos contatos, a apropriação do histórico familiar e social do paciente; a valorização das preocupações e queixas pode garantir um maior vinculo. Cabe lembrar, que as rotinas institucionais constantes são fundamentais no estabelecimento da confiança entre os terapeutas, usuários e comunidade. Assim, os registros dos atendimentos nos prontuários dão historicidade às intervenções com informações sobre os procedimentos realizados e a relação do usuário com os mesmos.

Portanto, o estabelecimento de protocolos complementares àqueles utilizados na ficha familiar e no Sistema de Informação na Atenção Básica - SIAB são importantes na notificação das demandas da população com deficiência. Essa tarefa pode ser realizada através de registros concisos, com dados sobre o desempenho no cotidiano, sobre a rede de relações familiares e sociais e com aspectos relacionados à participação social, de maneira que o entendimento das demandas desse usuário se amplie para além dos dados biomédicos.

Considerando que um dos atributos da APS é coordenar diversas ações e serviços essenciais para resolver necessidades mais freqüentes e mais complexas, a assistência reabilitacional da TO permite detectar as ações mais complicadas que necessitam de serviços especializados. Assim, elaborar pequenas adaptações, prescrever a melhor cadeira de rodas, inserir a comunicação alternativa ou confeccionar algum tipo de órtese é possível na UBS e até em alguns domicílios. No entanto, a elaboração de um assento ou encosto de cadeira de rodas digitalizadas, ações de dispensação de órteses confeccionadas com termoplásticos moldáveis a altas temperaturas, próteses ou consultas com especialistas, cirurgias, entre outras, devem ser encaminhados para outros níveis assistenciais e acompanhados no retorno à APS.

Assim, o TO pode e deve ser um facilitador da longitudinalidade nos cuidados em saúde das pessoas com deficiência e/ou incapacidades ao ampliar o leque de ofertas de procedimentos na UBS. Ao mesmo tempo, considerando os diferentes momentos da vida e do processo saúde-doença desse usuário, a TO pode desenvolver ações ligadas à continuidade da assistência encaminhando-o e/ou recebendo-o de outros níveis assistenciais.

As formas de atendimento em TO são diversas e ocorrem de acordo com a demanda dos usuários, quer na UBS, no domicilio ou em espaços da comunidade, através de cuidados individuais, grupais, dos familiares e de outras pessoas do circuito relacional.

Vale ressaltar que a atividade da TO na APS necessita respeitar a lógica de que o usuário é primeiramente acolhido pelas equipes de saúde, para posteriormente serem inseridos no programa de intervenções da área. A eleição da demanda e das prioridades é decidida pelas equipes em conjunto com os terapeutas ocupacionais e, subsecutivamente, incluídas em processos de planejamento do trabalho da UBS/ESF.

$\mathrm{Na}$ relação com os outros profissionais, a TO pode promover diversas ações de matriciamento com temas relacionados ao desempenho funcional nas atividades da vida cotidiana, participação, independência e autonomia, discussões sobre o que é deficiência e incapacidades, possibilidades de intervenção no domicilio, na comunidade, entre outros.

As atividades de matriciamento realizado pela TO são fundamentais no desenvolvimento de projetos terapêuticos singulares, pois, as trocas constantes entre os profissionais proporcionam uma maior capacidade dos serviços em responder, com qualidade, as necessidades dos usuários, além de fomentar a integralidade. Assim, a introdução da problemática da população que necessita de TO nas reuniões das equipes de saúde contribui para o processo de responsabilização pelo "caso".

Aqui se entende "caso" como uma situação 
exemplar na intervenção, que pode estar relacionada a questões específicas de um sujeito, como, por exemplo, a sua adaptação ao uso de equipamentos de Tecnologia Assistiva, como pode referir-se a problemas relacionais ou mesmo de participação social, como situações de inclusão escolar ou de outra natureza.

Desse modo, o trabalho da TO na APS está inserido numa proposta de condução continua do "caso" pela equipe de referência (CAMPOS, 1999), que pressupõe o entrelaçamento de ações preventivas, educativas, curativas e reabilitadoras no estabelecimento de desenhos de projetos terapêuticos, os quais são executados por essa mesma equipe e por um conjunto mais amplo de trabalhadores da saúde da APS e de outros níveis assistenciais, quando necessário.

\section{CONSIDERAÇÕES FINAIS}

De acordo com as premissas - acesso, longitudinalidade - vislumbram-se possibilidades impares da TO na APS. Entende-se também que essas premissas garantem a integralidade, a equidade e a universalização da atenção em saúde e que a TO tem um papel fundamental na efetivação das mesmas.

Nessa perspectiva, as práticas da TO na APS assumem o caráter prioritário de cuidados em saúde e sua atuação deve ser pautada pela compreensão dos processos saúde-doença que consideram as condições territoriais, sociais, biológicas e psicológicas e de que os usuários podem ser protagonistas na produção de saúde.

Outro aspecto a ser considerado é que a população não distingue os profissionais da reabilitação dos outros profissionais, entende que se trata de uma mesma equipe de saúde, o que eles desejam é o acesso e buscam a integralidade na assistência, evidenciando assim, a necessidade de um trabalho de TO integrado com os outros profissionais.

Isto posto, pode-se considerar que a TO, nesse nível assistencial, possui dinâmicas e cotidianos específicos, dotados de particularidades a serem consideradas em cada território, o que promove permanentes desafios na implementação das suas ações em saúde e evidencia a necessidade de reflexões sobre as suas possibilidades na APS.

ROCHA, E. F.; SOUZA, C. C. B. X. Occupational Therapy in Rehabilitation in Primary Health Care: Challenges and opportunities. Rev. Ter. Ocup. Univ. São Paulo, v. 22, n. 1, p. 36-44, jan./ abr. 2011.

\begin{abstract}
The goal of this paper is to contribute with ideas that may help to strengthen the actions Occupational Therapy (OT) has been able to develop in Primary Health Care (PHC) in the field of rehabilitation of people with disabilities. This proposition is based on both literature review and on the practical experience of REATA- Laboratory for Studies in Rehabilitation and Assistive Technology in the period from 1999 to 2010. We took into consideration the existing care models, Selective and Broad PHC, and the nature of these practices within the historical and practical-organizational context. Next, we describe the experiences developed by REATA in the PHC, which motivated the presentation of these ideas as an OT work proposition. We advocate the need for a shift from the occupational therapeutic interventions instituted under standardized, biomedical framework, or built within the public health hygienist design; towards the establishment of actions/strategies that address holistic aspects of the subjects and the territory, and with light technology adequate for the demands, as foreseen by the Broad PHC. Finally, we present possible OT contributions regarding their technological potential based on the premise that actions from this profession can contribute in promoting access and long-term health care and thus assist in the completeness and equity within the Basic Health System (SUS).
\end{abstract}

KEY WORDS: Occupational therapy; Primary health care; Family health; Nucleuar family; Rehabilitation; Disabled persons/rehabilitation. 


\section{REFERENCIAS}

BRASIL. Ministério da Saúde. Secretaria de Atenção à Saúde, Departamento de Atenção Básica. Política nacional de atenção básica. Brasília, 2006. (Série Pactos pela Saúde).

BRASIL. Ministério da Saúde. Portaria GM 154 de 24 de janeiro de 2008 cria os NASFs - Núcleo de Atenção Integral à Saúde da Família. Brasil, 2008.

BUENO, W. S.; MERHY, E. E. Os equívocos da NOB 96: uma proposta em sintonia com os Projetos Neoliberalizantes? 1997. Disponível em: <http://www.uff.br/saudecoletiva/professores/ merhy/artigos-14.pdf>. Acesso em: 02 mar. 2011.

CAMPOS, G. W. S. Equipes de referência e apoio especializado matricial: uma proposta de reorganização do trabalho em saúde. Ciência e Saúde Coletiva, v. 4, n. 2, p.393-404, 1999.

CUETO, M. The origins of primary health care and selective primary health care. American Journal of Public Health, v. 94, n. 11, p.1864 -1874, 2004.

CUNHA, E. M. Vinculo Longitudinal na Atenção Primária: avaliando os modelos assistenciais do SUS. $149 \mathrm{f}$. Tese de doutorado (Ciências na área de Saúde Pública) - ENSP/ FIOCRUZ, Rio de Janeiro, 2009.

ESCOREL, S. Reviravolta na saúde: origem e articulação do movimento sanitário. Rio de Janeiro: Fiocruz, 1999.

GIL, C.R.L. Atenção primária, atenção básica e saúde da família: sinergias e singularidades do contexto brasileiro. Cadernos de Saúde Pública, v. 22, n. 6, p. 1171-1181, 2006.

GIOVANELLA, L.; MENDONÇA, M. H. M. Atenção primária à saúde. In: GIOVANELLA, L.; ESCOREL, S.; LOBATO, L. V. C.; NORONHA, J. C.; CARVALHO, A. L. Políticas e sistema de

Recebido para publicação: 17/03/11

Aceito para publicação: 22/04/11 saúde no Brasil. Rio de Janeiro: FIOCRUZ, 2008. p.575-625.

MELO, R. J. Atenção primária à saúde e atenção básica à saúde: os discursos ideo-políticos do Ministério da Saúde. 2009. 209f. Dissertação (mestrado em Política Social) - Universidade Federal do Espírito Santo, Vitória, 2009.

ORGANIZAÇÃO PAN-AMERICANA DA SAÚDE, Organização Mundial da Saúde. Renovação da atenção primária nas américas. Documento de posicionamento da Organização Pan-Americana da Saúde. Washington: PAHO/WHO, 2005.

PAIM, J. S.; ALMEIDA FILHO, N. Saúde coletiva: uma "nova saúde pública" ou campo aberto a novos paradigmas. Rev. Saúde Pública, v. 32, n. 4, p. 299-316, 1998.

ROCHA, E. F. Reabilitação de pessoas com deficiência: a intervenção em discussão. São Paulo: Roca, 2006.

ROCHA, E. F., KRETZER, M. R. Avaliação da implantação das ações de reabilitação no Programa de Saúde da Família (PSF) da Fundação Zerbini e Secretaria Municipal de Saúde de São Paulo - Região Sudeste - Sapopemba/Vila Prudente - período 2000/2006. Relatório Técnico - Edital MCT-CNPq / MS-DAS/ SAS - No 49/2005; Processo nº 402234/05-7. 2008.

ROCHA, E. F.; KRETZER, M. R. Ações de reabilitação de pessoas com deficiência na estratégia da saúde da família da Fundação Zerbini e Secretaria Municipal de Saúde de São Paulo - Região Sudeste - Sapopemba/Vila Prudente - período 2000/2006. Revista de Terapia Ocupacional da Univ. São Paulo, v. 20, n. 1, p. 5967, 2009.

STARFIELD, B. Atenção primária: equilíbrio entre necessidades de saúde, serviços e tecnologia. Brasília: UNESCO, Ministério da Saúde, 2002. 